\section{Catherine Pavoine Françoise Pecker}

\title{
Lumière sur le calcium
}

La mesure directe du calcium libre dans les cellules vivantes a commencé dans les années 1960 avec l'aequorine, une protéine bioluminescente sensible au calcium. Le clonage du gène de l'aequorine a remis cette molécule à la mode car les techniques de biologie moléculaire permettent son utilisation pour la mesure du $\mathrm{Ca}^{2+}$ dans les organites cellulaires. Le développement d'indicateurs fluorescents de synthèse, de première (Quin2) et de deuxième génération (Fura2, Indol, Fluo3, Fura red, etc.), tous dérivés de l'EGTA, couplé aux progrès de la microscopie et du traitement informatisé des images, rend possible aujourd'hui l'étude cinétique des variations du $\mathbf{C a}^{2+}$ dans une cellule unique et ses divers compartiments, ou dans une population cellulaire, au repos ou en réponse à une stimulation. Ce domaine est à l'heure actuelle l'un des plus évolutifs de la biologie cellulaire.

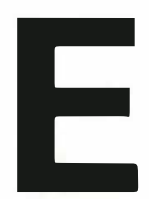

n 1882, Ringer découvrait le rôle du calcium dans la contraction musculaire par la simple comparaison des effets de l'eau du robinet et de l'eau distillée. Il est apparu depuis que de nombreuses actions hormonales, mettant en jeu l'activation de systèmes enzymatiques dépendants du $\mathrm{Ca}^{2+}$, sont relayées par des élévations du $\mathrm{Ca}^{2+}$ cytosolique libre. Le contrôle rigoureux de l'homéostasie calcique intracellulaire, dont les mécanismes ont été détaillés dans deux revues précédentes de médecine/sciences [1, 2], est essentiel à ce rôle de messager de l'ion $\mathrm{Ca}^{2+}$ ainsi qu'à la survie cellulaire. Le développement récent d'indicateurs du $\mathrm{Ca}^{2+}$ libre fluorescents, associé aux progrès technologiques de l'informatique et de l'ima- gerie, permet aujourd'hui la mesure directe des variations de $\mathrm{Ca}^{2+}$ intracellulaire sur les cellules vivantes. Comme en a témoigné la réunion du Calcium Club, à Milan (20-25 septembre 1993), ce domaine est à l'heure actuelle l'un des plus évolutifs et des plus exaltants de la biologie cellulaire.

\section{L'aquorine, une protéine bioluminescente spécifique du $\mathrm{Ca}^{2+}$}

Les premières mesures du taux de $\mathrm{Ca}^{2+}$ cytosolique libre ont été réalisées par Ridgway et Ashley en 1967, à l'aide d'une photoprotéine, l'aequorine, sur des cellules musculaires géantes. L'aequorine a été découverte et isolée de la méduse Aequora victoria en 1962 par Shimo- 


\section{RÉFÉRENCES}

1. Mauger JP, Claret M. Flux de calcium et contrôle de la glycogénolyse hépatique. méderine/sciences $1987 ; 3$ : 574-81.

2. Bismuth G, Trautmann A, Debré P. Calcium et activation lymphocytaire T. méde cine/sciences $1990 ; 6$ : 762-9.

3. Blinks JR, Wier WG, Hess P, Prendergast FG. Measurement of $\mathrm{Ca}^{2+}$ concentration in living cells. Prog Biophys Mol Biol $1982 ; 40: 1-114$.

4. Cobbold PH, Rink TJ. Fluorescence and bioluminescence measurement of cytoplasmic free calcium. Biochem J 1987; 248: 31328.

5. Cobbold PH, Bourne PK. Aequorin measurements of free calcium in single heart cells. Nature $1984 ; 312$ : 444-6.

6. Tsien RY, Adams SR. Measurement and manipulation of cellular $\mathrm{Ca}^{2+}$. Neuroscience Facts 1993; 4 : 14-6.

7. Llinas R, Sugimori M, Silver RB. Microdomains of high calcium concentration in a presynaptic terminal. Science 1992; 256 : $677-9$.

8. Rizzuto R, Simpson AWM, Brini M, Pozzan $T$. Rapid changes of mitochondrial $\mathrm{Ca}^{2+}$ revealed by specifically targeted recombinant aequorin. Nature $1992 ; 358$ : 325-7.

9. Kendall JM, Dormer RL, Campbell AK. Targeting aequorin to the endoplasmic reticulum of living cells. Biochem Biophys Res Commun 1992; 189: 1008-16.

10. Tsien RY, Pozzan T, Rink TJ. Calcium homeostasis in intact lymphocytes : cytoplasmic free calcium monitored with a new, intracellularly trapped fluorescent indica-

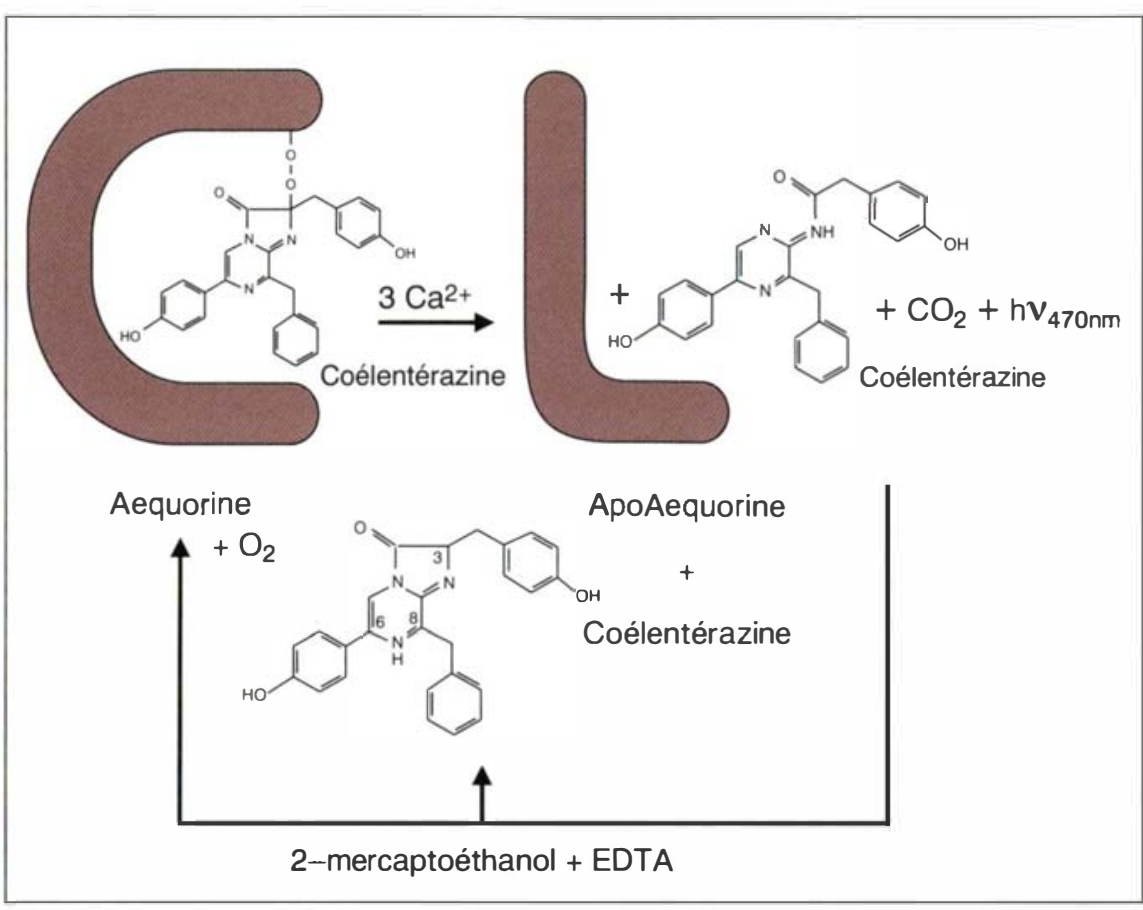

Figure 1. Réaction de bioluminescence de l'aequorine. L'æquorine est constituée d'une apoprotéine de $20 \mathrm{kDa}$, étroitement associée à un chromophore de 423Da, la coélentérazine. La liaison de trois ions $\mathrm{Ca}^{2+}$ à une molécule d'æquorine déclenche l'oxydation de la coélentérazine en coelentéramide, sa dissociation de l'apoæquorine ainsi que la libération de $\mathrm{CO}_{2}$, et s'accompagne d'une émission transitoire de photons à $470 \mathrm{~nm}$ qui peut être captée et traduite en signal électrique par un photomultiplicateur.

mura, qui l'a proposée comme indicateur calcique dès 1963 (pour des revues, consulter: [3] et [4]) (Tableau I). L'aequorine est constituée d'une apoprotéine de $20 \mathrm{kDa}$, étroitement associée à un chromophore de $423 \mathrm{Da}$, la coélentérazine. La liaison de trois ions $\mathrm{Ca}^{2+}$ à une molécule d'aequorine déclenche l'oxydation de la coélentérazine, sa dissociation de l'apozquorine ainsi que la libération de $\mathrm{CO}_{2}$, et s'accompagne d'une émission transitoire de photons à $470 \mathrm{~nm}$ qui peut être captée et traduite en signal électrique par un photomultiplicateur. Cette réaction de bioluminescence aboutit à l'extinction de la molécule d'aequorine, du moins en l'absence de coélentérazine réduite, qui est nécessaire à la régénération de la photoprotéine (figure 1).
Parmi les avantages de cet indicateur calcique, on peut noter la relative rapidité de la cinétique de la réaction de bioluminescence stimulée par le $\mathrm{Ca}^{2+}$, qui a permis la détection des variations $\mathrm{du}^{\mathrm{Ca}^{2+}}$ au cours du cycle cardiaque [5]. En outre, la taille de la protéine ainsi que l'existence de nombreuses charges négatives favorisent sa localisation dans le cytosol et rendent improbable sa séquestration dans les organites intracellulaires ou son relargage à l'extérieur de la cellule, ce qui permet des expériences de plusieurs heures.

L'une des difficultés majeures rencontrées lors de l'utilisation de l'æequorine concerne l'interprétation des signaux lumineux émis par la sonde en termes de concentrations absolues de $\mathrm{Ca}^{2+}$ : la quantification des résultats nécessite, en effet, 
d'une part l'établissement de courbes de calibration réalisées in vitro, d'autre part la détermination de la concentration intracellulaire de la sonde en fin de chaque expérience. Le premier point suppose l'utilisation de tampons qui doivent reproduire au mieux les conditions de milieu intracellulaire en termes de $\mathrm{pH}$, force ionique, viscosité et composition ionique. En second lieu, il est nécessaire, en fin d'expérience, de déterminer la bioluminescence maximale des cellules (Lmax). Cela requiert une lyse rapide des cellules et l'exposition des molécules d'aequorine à une concentration maximale de $\mathrm{Ca}^{2+}$. A partir de la courbe de calibration $(\mathrm{L} / \mathrm{L} \max )=$ $\mathrm{f}\left(\log \left(\mathrm{Ca}^{2+}\right.\right.$ libre $\left.)\right)$, établie in vitro à l'aide de tampons Ca/EGTA, la mesure des deux intensités de fluorescence, $\mathrm{L}$ et Lmax, permet de déduire la concentration de $\mathrm{Ca}^{2+}$ libre.

De fait, la grande majorité des études réalisées avec de l'aequorine se sont cantonnées à des analyses qualitatives. Il faut aussi remarquer que l'intensité de bioluminescence de l'aequorine ne se rapporte pas seulement à la concentration de $\mathrm{Ca}^{2+}$ mais qu'elle est aussi fonction de la concentration de la photoprotéine. Cela interdit l'étude du calcium à l'échelle intracellulaire, la concentration d'aequorine pouvant être différente selon l'épaisseur du champ, du centre à la périphérie de la cellule, et ne permet pas non plus le suivi de la propagation de vagues de Ca.

D'autres limitations à l'utilisation de l'aequorine sont rencontrées. D'une part, la vitesse de la réaction de bioluminescence dépend directement de la concentration de $\mathrm{Ca}^{2+}$, ce qui impose un seuil inférieur à la détection de l'ion de l'ordre de $0,1 \mu \mathrm{M}$. D'autre part, il est nécessaire d'injecter des doses suffisamment élevées de la photoprotéine pour obtenir un signal détectable en présence de faibles concentrations de $\mathrm{Ca}^{2+}$. Enfin, l'utilisation de l'aequorine nécessitant à l'origine sa microinjection, cette technologie a longtemps été limitée à l'étude des cellules d'invertébrés de grande taille et réservée aux laboratoires spécialisés dans la physiologie des cellules excitables, familiarisés avec la technique de micro-injection et disposant d'un équipement de pointe dans les domaines de l'optique et de l'électronique.

Le clonage récent du gène de l'apoaequorine et la synthèse chimique de la coélentérazine ont ouvert de nouveaux horizons à l'utilisation de cette sonde ([6], $m / s \quad n^{\circ} 1$, vol. 10, p. 123). La transfection du gène dans des cellules en culture ainsi que la régénération du signal de bioluminescence par incubation des cellules transfectées avec de la coélentérazine permettent aujourd'hui d'obtenir des cellules qui, sans le stress de la micro-injection ou des méthodes de perméabilisation membranaire, expriment des quantités de photoprotéine suffisantes à la détection de signaux sur cellules uniques de petite taille et donnent également accès à des études sur populations cellulaires.

En outre, des modifications chimiques de la coélentérazine ont permis de déplacer la courbe d'activation de la bioluminescence de l'aquorine vers des concentrations de calcium plus élevées et de détecter des domaines intracellulaires à très haute concentration de calcium, ou hotspots, de l'ordre de $0,1 \mathrm{mM}$ [7]. Enfin, l'addition de séquences spécifiques

Tableau I

LES SONDES CALCIQUES BIOLUMINESCENTES ET FLUORESCENTES UTILISÉES DANS LA LITTÉRATURE

\begin{tabular}{|c|c|c|c|c|c|c|}
\hline Réaction & Sonde & Réf. & Excitation & $\lambda \operatorname{Ex}(\mathrm{nm})$ & $\lambda \operatorname{Em}(\mathrm{nm})$ & $\mathrm{Kd} \mathrm{Ca}^{2+}(\mathrm{nM})^{\mathrm{a}}$ \\
\hline
\end{tabular}

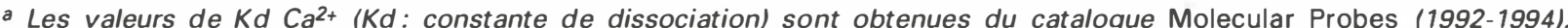

b $\lambda$ d'émission maximale de la sonde liée au $\mathrm{Ca}^{2+}+\lambda d^{\prime} e^{\prime}$ ission maximale de la sonde libre.

c $\lambda$ d'émission maximale du fluo3 lié au $\mathrm{Ca}^{2+} / \lambda$ d'émission maximale du Fura red libre.

d L'aequorine lie $3 \mathrm{Ca}^{2+}$; une $\mathrm{Ec}_{50}$ (concentration de $\mathrm{Ca}^{2+}$ pour laquelle on obtient la demi-saturation de la sonde) de $3 \mu \mathrm{M}$ a été déterminée. 


\section{RÉFÉRENCES}

11. Tsien R, Pozzan T. Measurement of cytosolic free $\mathrm{Ca}^{2+}$ with Quin 2. Methods Enzymol 1989; 172: 23()-62.

12. Grynkiewicz (; Poenie M, Tsien RY: A new generation of $\left(\mathrm{C}^{2+}\right.$ indicators with greatly improved fluorescence properties. f Biol (hem 1985; 260: 3440-50.

13. Roe MW, Lcmasters IJ, Herman B. Assessment of Fura-2 for measurements of cytosolic free calcium. (iell Calcium 1990); $11: 63-73$.

14. Poenie M. Alteration of intracellular Fura-2 fluorescence by viscosity. A simple correction. Cell Calcium 1990); 11 : 85-91.

15. Hove-Madsen I., Bers IDM. Indo-l binding to protein in permeabilized ventricular myocytes alters its spectral and $\mathrm{Ca}$ binding properties. Biophys / 1992; 63 : 89-97.

16. Theler JM, Mollard P, Guérineau N, Vacher P, Pralong W'F, Schlegel W', W'ollheim (:B. Video imaging of cytosolic ( $\mathrm{ad}^{2+}$ in pancreatic $\beta$-cells stimulated by glucose, carbachol and ATP. / Biol Chem 1992; 267 : 18110-7.

17. L'to A, Arai H, Ogawa Y.. Reassessment of Fura-2 and the ratio method for determination of intracellular $\mathrm{Ca}^{2+}$ concentrations. Ciell Calcium 1991; 12: 29-37.

18. Malgaroli A, Milani D, Meldolesi J, Pozaan T. Fura-2 measurement of cytosolic free $\mathrm{Ca}^{2+}$ in monolayers and suspensions of various types of animal cells. I (ell Biol 1987; 105 : 2145-55.

19. Di Virgilio F, Steinberg TH, Silverstein SC. Inhibition of Fura-2 sequestration and secretion with organic anion transport blockers. Ciell Calrium 1990); 11: 57-62.

20. Minta A, Kao JPY, Tsien RY. Fluorescent indicators for cytosolic calcium based on rhodamine and fluorescein chromophor res. I Biol Chem 1989; 264: 8171-8.

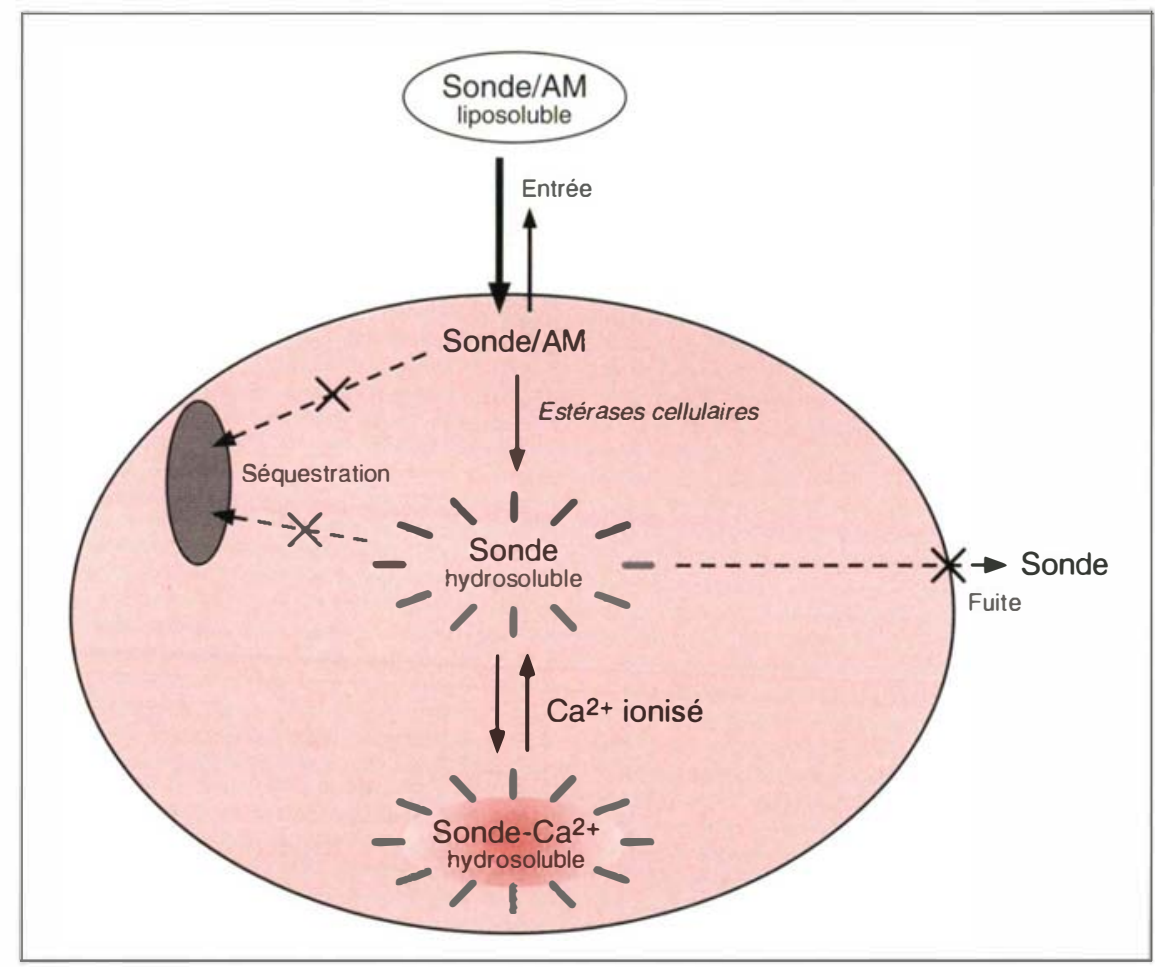

Figure 2. Charge d'une cellule en sonde IAM. Le dérivé acétoxyméthylé (sonde AM) de la sonde, lipophile, qui pénètre dans les cellules est hydrolysé par les estérases intracellulaires en sonde hydrosoluble qui se trouve piégée dans la cellule. Ellipse grise: organites intracellulaires.

au gène de l'apozequorine a permis de cibler la localisation subcellulaire de la protéine exprimée vers les mitochondries [8] ou le réticulum endoplasmique [9]. Ces travaux apportent des domées nouvelles concernant l'homéostasie calcique: ils permettent de démontrer la compartimentation du calcium, de révéler les interactions entre les différents compartiments intracellulaires ainsi que leur contribution, en particulier lors d'une stimulation cellulaire hormonale.

Ainsi, la nouvelle orientation des recherches vers le domaine de la biologie moléculaire a suscité un important regain d'intérêt pour cette vieille molécule. La prochaine étape de cette révolution concerne sans nul doute l'établissement de lignées d'animaux transgéniques exprimant le gène de l'apoaquorine dans la plupart de leurs tissus...

\section{Les sondes fluorescentes de première génération: le Quin2}

Au début des années 1980, l'équipe de Tsien a développé le Quin2 (Tableau I et figure 3), dérivé fluorescent du chélateur classique du $\mathrm{Ca}^{2+}$ l'EGTA, qui lie le $\mathrm{Ca}^{2+}$ avec une stoechiométrie de $1: 1$ ([10] et pour des revues: [4] et [11]). Ce nouveau type d'indicateur calcique, constitué d'un fluorophore associé au chélateur du $\mathrm{Ca}^{2+}$, est bien plus qu'un simple marqueur fluorescent indiquant la présence du $\mathrm{Ca}^{2+}$ puisque la liaison de l'ion entraîne des modifications de l'intensité de la fluorescence émise $\left(\mathrm{F}_{\mathrm{enn}}\right)$ par le fluorophore, ce qui permet d'établir des courbes de calibration $F_{e m}=f$ $\left[\mathrm{Ca}^{2+}\right]$ (Tableau I et figure 3).

Cette même équipe a également eu l'idée astucieuse de transformer chi- 
miquement la molécule de Quin2 en un dérivé acétoxyméthylé, lipophile, le Quin2-AM, ce qui a simplifié extraordinairement les protocoles de charge et permis d'accéder à l'étude de cellules de petite taille. Ia charge des cellules en Quin2-AM se fait par simple préincubation en présence de la sonde-AM qui pénètre (plus ou moins) facilement dans les cellules et est hydrolysée par les estérases intracellulaires en Quin2 hydrophile qui se trouve piégé dans la cellule (figure 2). Le Quin2-AM est une molécule faiblement fluorescente qui émet à $440 \mathrm{~nm}$. Le déplacement de ce pic d'émission de fluorescence de $440 \mathrm{~nm}$ pour Quin2-AM vers $492 \mathrm{~nm}$ pour Quin2 est utilisé comme test de l'hydrolyse du Quin2-AM en Quin2 libre.

Le Quin2 présentait encore de nouveaux avantages. D'une part, son affinité forte pour l'ion $\mathrm{Ca}^{2+}$, avec une constante de dissociation de l'ordre de $114 \mathrm{nM}$, permet de mesurer de façon précise de faibles variations de $\mathrm{Ca}^{2+}$, proches de la concentration intracellulaire de repos des cellules. D'autre part, sa liaison au $\mathrm{Ca}^{2+}$ est spécifique, non modi- fiée par les concentrations intracellulaires de $\mathrm{Mg}^{2+}$, et peu sensible aux variations physiologiques de $\mathrm{pH}$. Malgré l'intérêt indéniable que présentait à l'époque cette nouvelle molécule, l'utilisation de lumière ultraviolette (UV) pour l'excitation du Quin2 $(340 \mathrm{~nm})$ a très certainement retardé la généralisation de son utilisation. En effet, cela condamnait l'emploi de systèmes optiques en verre, qui absorbent quasi totalement les $\lambda<350 \mathrm{~nm}$, et exigeait un équipement optique onéreux, en quartz. Par ailleurs, l'intensité de fluorescence émise par une sonde, $F_{\mathrm{cm}}$, dépend de son rendement quantique $\left(\mathrm{F}_{\mathrm{em}}=\mathrm{C} \cdot \mathrm{k} . \mathrm{I}_{\mathrm{exc}}\right)$ où C est la concentration de la sonde, $\mathrm{k}$ le rendement quantique et $\mathrm{I}_{\mathrm{cxc}}$ l'intensité de l'excitation. La détection sur cellule unique du Quin2, qui possède un faible rendement quantique, nécessite la charge d'un nombre important de molécules par cellule. Le risque est alors de tamponner le $\mathrm{Ca}^{2+}$ endogène et donc de modifier l'homéostasie calcique intracellulaire, ce qui peut conduire à des mesures artéfactuelles de variations de l'ion. En outre, le Quin2 est une molécule extrêmement photolabile dont la fluorescence s'éteint après absorption de 3 ou 4 photons en moyenne. Ce phénomène d'extinction (bleaching) limite nettement la durée des expérimentations. Du fait de sa taille, la molécule de Quin2 est également plus susceptible que ne l'était l'aequorine d'être expulsée hors de la cellule. Ces deux phénomènes compliquent la quantification des données qui nécessite en fin d'expérience, comme dans le cas de l'aequorine, une estimation de la quantité de sonde intégrée dans les cellules. La mesure sera d'autant plus erronée que les phénomènes d'extinction et de fuite de la sonde en cours d'expérience auront été importants. Enfin, outre son éventuelle nocivité, l'irradiation des cellules par des UV produit une autofluorescence cellulaire susceptible d'interférer avec le spectre de l'indicateur. De fait, ces deux inconvénients conditionnent l'utilisation de toute sonde fluorescente excitable dans l'UV.

Pour l'ensemble de ces raisons, l'utilisation du Quin2 a généralement été réservée à des études de popu-

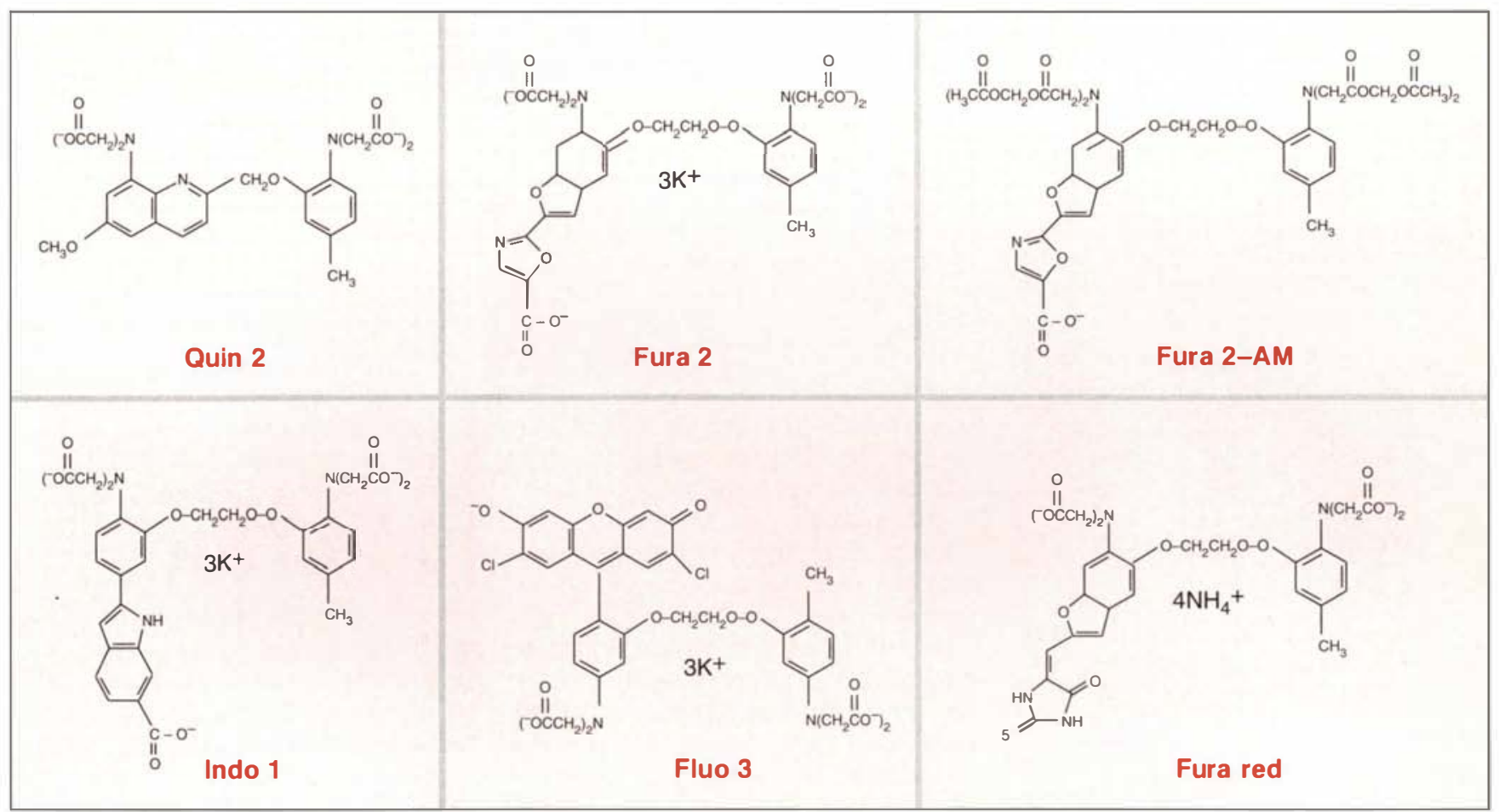

Figure 3. Structure des sondes calciques fluorescentes. 


\section{RÉFÉRENCES}

21. Haugland RP. Calcium indicators, chelators and ionophores. Molecular probes handbook of fluorescent probes and research che micals 1992-1994; Set 20: 113-28.

22. Lipp P, Niggli E. Ratiometric confocal $\mathrm{Ca}^{2+}$-measurements with visible wavelength indicators in isolated cardiac myocytes. (eell Calcium 1993; 14 : 359-72.

23. June CH, Rabinovitch PS. Flow cytometric measurement of intracellular ionized calcium in single cells with Indo-1 and Fluo-3. Methods Cell Biol 1990; 33 : 37-58.

24. Shotton D, White N. Confocal scanning microscopy: three dimensional biological imaging. Trends Biochem Sci 1989; 14: 435-9

25. Kaplan JH, Somlyo AP. Flash photolysis of caged compounds: new tools for cellular physiology. Trends Neurol Sri 1989; 12: $54-9$.

26. Berridge MJ. Inositol triphosphate and calcium signalling. Nature 1993; 361: 315-25.

27. Ruttner Z, Ligeti I, Reinlib I, Hines $\mathrm{K}$, McLaughlin AC. Monitoring of intracellular free calcium in perfused rat liver. (ell Calcium 1993; 14 : 465-72.

28. Knoll G, Grässle A, Braun C, Probst W, Höhne-Zell B, Plattner H. A calcium influx is neither strictly associated with nor necessary for exocytotic membrane fusion in Paramecium cells. Cell Calcium 1993; $14: 173-83$

29. Donnadieu E, Bismuth G, Trautmann A. Calcium fluxes in T-lymphocytes. I Biol Chem 1992; 267: 25864-72.

30. Ogden DC, Khodakhah K, Gray PTA, Carter TD, Capiod T. Mechanisms of intracellular Ca release during hormone and neurotransmitter action investigated with flash photolysis. J Exp Biol 1993; 184 : 105-27.

31. Putney JW. Capacitative calcium entry revisited. Cell Calcium 1990; 11: 611-24.

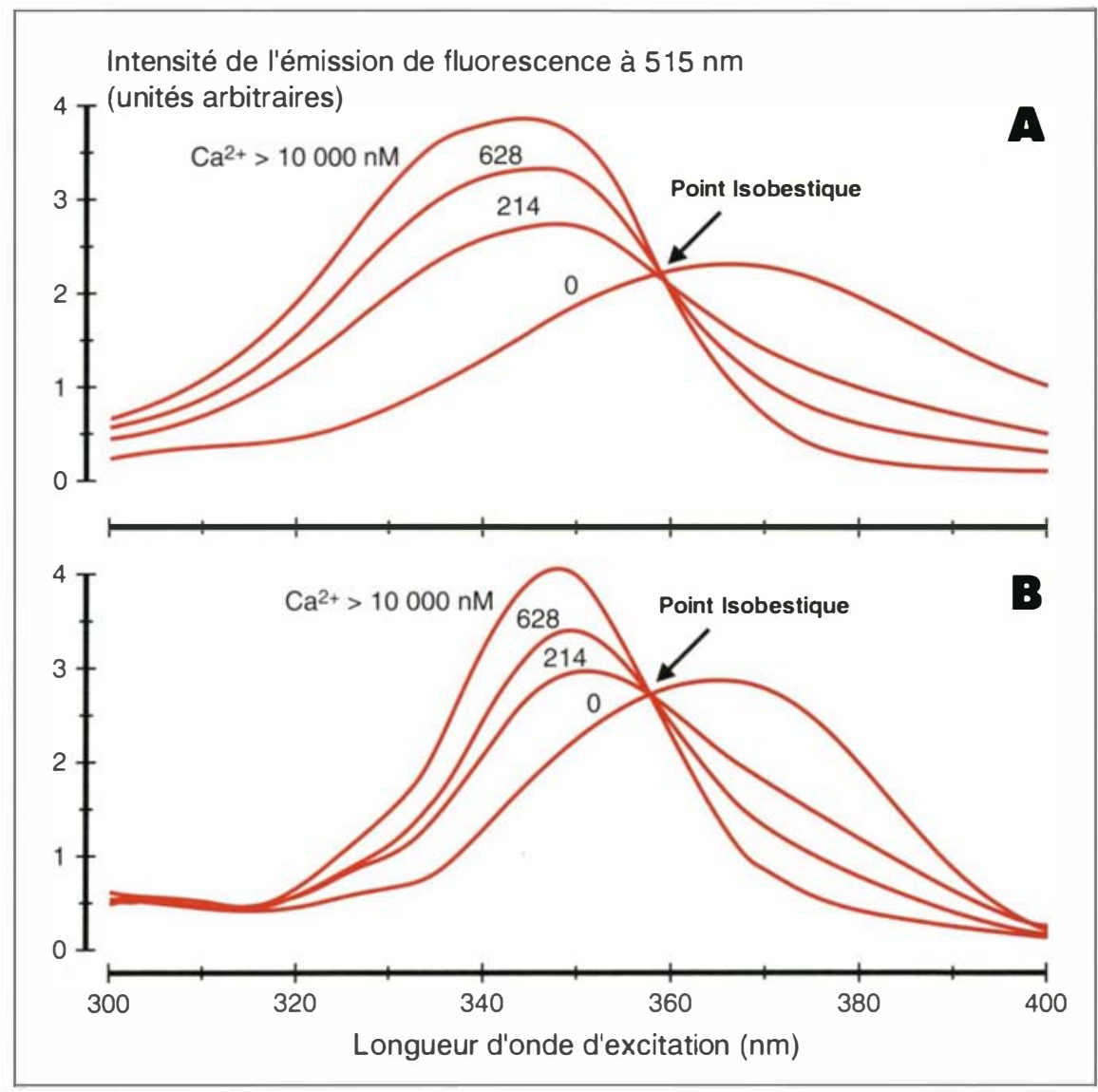

Figure 4. Spectres d'excitation du Fura2 à différentes concentrations de calcium. A. Mesures effectuées en cuvette de spectrofluorimètre. B. Mesures effectuées sur microscope à fluorescence. La liaison du calcium à cette sonde s'accompagne non seulement d'une variation de son intensité de fluorescence, mais aussi d'un déplacement du spectre d'excitation. La liaison du calcium à la sonde se traduit par une augmentation de la fluorescence émise à $515 \mathrm{~nm}$ pour les longueurs d'onde d'excitation inférieures à $360 \mathrm{~nm}$ corrélée à une diminution de la fluorescence émise pour les longueurs d'onde d'excitation supérieures à $360 \mathrm{~nm}$. Pour une excitation à $360 \mathrm{~nm}$, l'émission de fluorescence $F_{360}$ est indépendante de la présence de $\mathrm{Ca}^{2+}$ (point isobestique). La mesure du rapport des fluorescences $F_{340}$ et $F_{380}$ permet de calculer la concentration absolue de $\mathrm{Ca}^{2+}$, indépendamment de la concentration de la sonde [12].

lations de cellules réalisées en cuvette de spectrophotomètre, technique de photométrie du $\mathrm{Ca}^{2+}$. Cette approche est tout à fait indiquée pour établir des comparaisons directes avec des mesures biochimiques. En revanche, elle offre des résolutions quantitatives et temporelles limitées puisque plusieurs cellules ne répondront pas avec la même amplitude et de façon synchrone à un stimulus donné.

Aujourd'hui, le Quin2 est délaissé pour de nouvelles molécules.

\section{Les sondes de seconde génération: le Fura2 et I'Indo1, le Mag-Fura2 et le Mag-Indo1}

La synthèse par l'équipe de Tsien du Fura2 et de l'Indo1, tous deux également dérivés de l'EGTA, sous les deux formes, acide et acétoxyméthyl-estérifiée (AM), a constitué un progrès majeur [12] (Tableau I et figure 3). En référence au Quin2, que l'on peut qualifier de sonde 


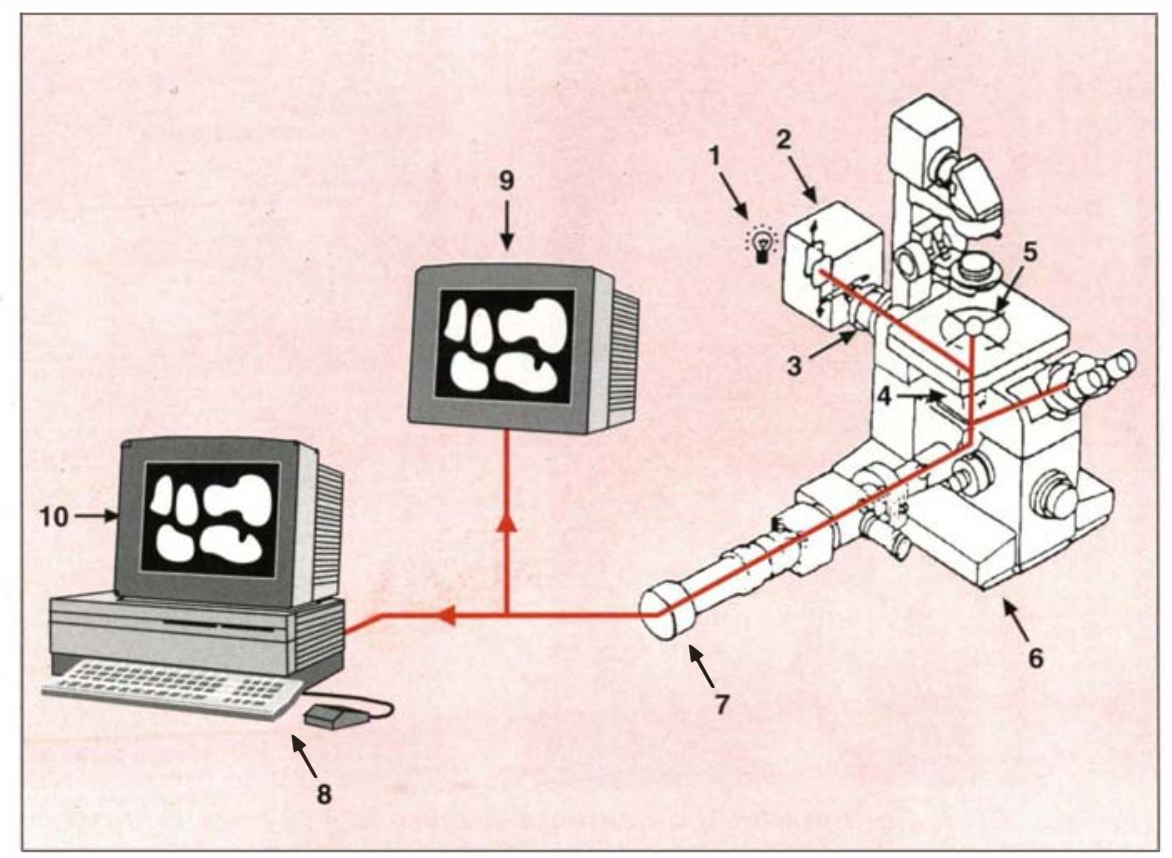

Figure 5. Spectrofluorimétrie avec traitements d'images. Appareillage de mesure du $\left[\mathrm{Ca}^{2+}\right]_{i}$ en double excitation-mono-émission. Le principe de l'appareil est le suivant: les cellules, chargées en sonde fluorescente sont placées sur la platine d'un microscope inversé. L'exposition, alternative et quasi simultanée, des cellules à deux longueurs d'onde d'excitation se fait grâce à un porte-filtres dont le mouvement est commandé par l'ordinateur. Les deux images de fluorescence, prises quasi-simultanément aux deux longueurs d'onde d'excitation et recueillies à une même longueur d'onde d'émission, sont visualisées à l'aide de la caméra. Elles sont transmises à l'ordinateur et leur rapport est calculé et traduit en une image pseudo-couleur qui est affichée à l'écran. 1: lampe xénon (100 W). 2: porte-filtres alternant les 2 filtres d'excitation. 3: filtre atténuateur. $4:$ miroir dichroïque. 5 : cellules. 6: microscope inversé. 7: caméra. 8: ordinateur: acquisition et traitement des images, stockage sur disques magnéto-optiques. 9 : écran de contrôle (image de fluorescence). 10: écran de l'ordinateur (image $\mathrm{Ca}^{2+}$ en pseudo-couleurs).

mono-excitation/ mono-émission, le Fura2 est une sonde double excitation/ mono émission et l'Indol une sonde mono excitation/ double émission. Cela signifie que la liaison du calcium à ces sondes s'accompagne non seulement d'une variation de leur intensité de fluorescence mais aussi d'un déplacement du spectre d'excitation (Fura2) (figure 4) ou du spectre d'émission (Indol). L'émission de fluorescence du Fura2 à $515 \mathrm{~nm}$ est mesurée en réponse à une excitation à $340 \mathrm{~nm}\left(\mathrm{~F}_{340}\right)$ puis à $380 \mathrm{~nm}\left(\mathrm{~F}_{380}\right)$ (figures 4 et 5$)$, et si $\mathrm{m} / \mathrm{s} n^{\circ} 4$ vol. 10, avil 94
$\beta=\mathrm{F}_{380}$ en absence de $\mathrm{Ca}^{2+} / \mathrm{F}_{380}$ à concentration saturante de $\mathrm{Ca}^{2+}$; $\mathrm{R}=\mathrm{F}_{340} / \mathrm{F}_{380}$;

$\mathrm{Rmin}=\mathrm{R}$ en absence de $\mathrm{Ca}^{2+}$; $\mathrm{Rmax}=\mathrm{R}$ à concentration saturante de $\mathrm{Ca}^{2+}$; en rappelant que $\mathrm{F}=$ C.k.I ${ }_{\text {exc. }}$.

Les paramètres de calibration Rmin, Rmax et Kd. $\beta$ sont définis pour chaque condition expérimentale en tenant compte de l'intensité de la lampe, de l'utilisation d'un filtre atténuateur, des réglages de la caméra et de l'objectif du microscope... On notera que les protocoles de calibration préfèrent aujourd'hui l'utilisation de ionophores calciques, tels le 4-Br A23187 ou la ionomycine, à la lyse cellulaire. La sonde Indol est aussi utilisée en mesurant un rapport de valeurs de fluorescence, mais, contrairement au Fura2, l'excitation se fait à une seule longueur d'onde $(355 \mathrm{~nm})$ et la fluorescence émise est suivie à deux longueurs d'onde (405 et $485 \mathrm{~nm}$ ). Dans la formule de Grynkiewicz:

$\beta=F_{485}$ en l'absence de $\mathrm{Ca}^{2+} / \mathrm{F}_{485}$ à concentration saturante de $\mathrm{Ca}^{2+}$;

$$
\mathrm{R}=\mathrm{F}_{405} / \mathrm{F}_{485} \text {. }
$$

L'utilisation des sondes à deux longueurs d'onde présente l'avantage majeur de pouvoir évaluer les concentrations absolues de $\mathrm{Ca}^{2+}$ sans qu'il soit nécessaire de quantifier systématiquement, à la fin de chaque expérience, la concentration de la sonde dans la cellule. En outre, ce mode de calcul affranchit des problèmes de fuite ou d'extinction de la sonde au cours de l'expérience. A ce propos, il faut noter que le Fura2 est une molécule relativement photorésistante. Cependant, cette technique ne met pas pour autant à l'abri de certains artéfacts d'estimation du taux de $\mathrm{Ca}^{2+}$ puisque des variations de viscosité, de force ionique ou de liaison de la sonde à des protéines intracellulaires en cours d'expérience modifient le $\mathrm{Kd}$ de la sonde pour le $\mathrm{Ca}^{2+}[13-15]$. Le Kd du Fura2 varie de $225 \mathrm{nM}$ à $760 \mathrm{nM}$ lorsque la force ionique augmente de 0,1 à 0,23 .

Le Fura2 et l'Indol ont un fort rendement quantique et un signal de fluorescence intense peut être obtenu sur une cellule unique pour 
une concentration de sonde suffisamment faible $(<50 \mu \mathrm{M})$ pour ne pas interférer avec les mécanismes cellulaires de régulation du $\mathrm{Ca}^{2+}$. En outre, seules les sondes utilisées à deux longueurs d'onde d'émission ou d'excitation permettent l'analyse quantitative de variations locales de $\left[\mathrm{Ca}^{2+}\right]_{i}$ et de vagues calciques intracellulaires. Ces qualités font de ces deux sondes des indicateurs calciques de choix pour les études d'imagerie sur cellules uniques en microscopie (technique d'imagerie du $\mathrm{Ca}^{2+}$ ) [16] (figures 7 et 8).

L'utilisation, en imagerie, des indicateurs calciques dont les spectres d'excitation sont localisés dans l'UV a nécessité la maîtrise de plusieurs difficultés. En effet, l'absorption très importante des longueurs d'onde UV $<350 \mathrm{~nm}$ par les objectifs de microscope ne permet pas toujours l'utilisation de ces sondes aux longueurs d'onde définies en cuvette de quartz dans un spectrophotomètre $[14,17]$ (figure 4). En ce qui concerne le Fura2, plusieurs types de compromis ont été adoptés selon les laboratoires: l'abandon de la longueur d'onde d'excitation $340 \mathrm{~nm}$ en faveur de longueurs d'onde mieux transmises $-350 \mathrm{~nm}$ ou $360 \mathrm{~nm}$-, l'atténuation de $\mathrm{F}_{380}$ par l'interposition d'un filtre atténuateur devant le filtre d'excitation $380 \mathrm{~nm}$, ou le choix d'une longueur d'onde d'excitation de $385 \mathrm{~nm}$ ou $390 \mathrm{~nm}$ au lieu de $380 \mathrm{~nm}$ afin d'obtenir deux intensités d'émission compatibles, $F_{340}$ et $\mathrm{F}_{385}$ ou ${ }_{390}$. De même, les longueurs d'onde choisies pour les études en Indol diffèrent selon les laboratoires et les appareils utilisés.

Pour chaque type cellulaire et chaque type de sonde utilisés, il est nécessaire d'établir empiriquement les conditions optimales de charge [18] : la pénétration des sondes dans les cellules a été améliorée par l'utilisation de molécules "dispersantes» comme des détergents ou des protéines du sérum. De nombreuses études témoignent également des risques de compartimentation de ces sondes dans des organites différentes selon le type cellulaire concerné [19]; ces compartiments, identifiés par colocalisation avec des marqueurs fluorescents spécifiques, sont le réticulum, les mito-

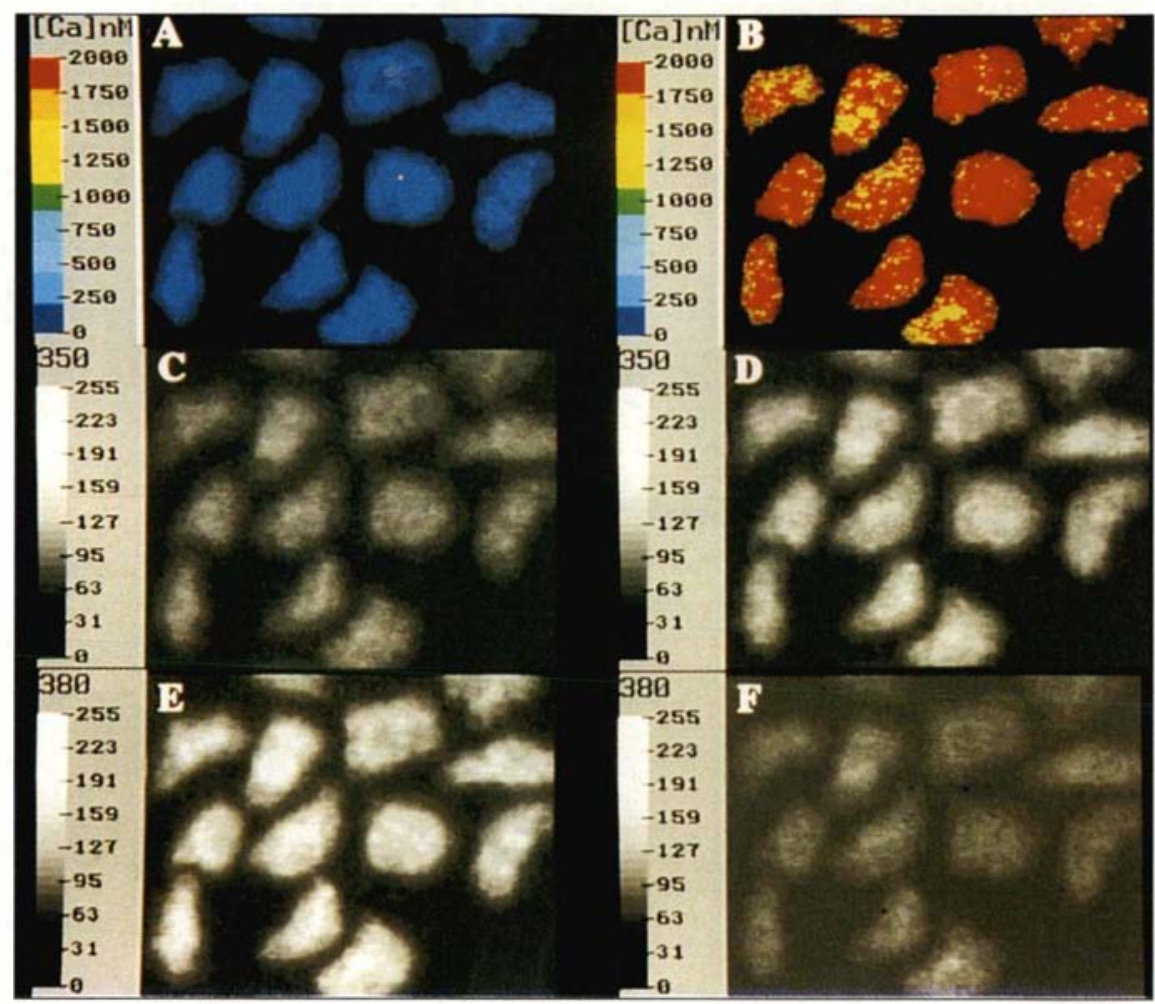

Figure 6. Effet du ionophore calcique 4-Bromo-A23187 sur la concentration du calcium cytosolique libre $\left(\left[\mathrm{Ca}^{2+}\right]_{i}\right)$ de cellules de ventricules de cour d'embryons de poulet de 14 jours, chargées en Fura 2. Deux temps sont représentés, $t=0(A, C, E)$ et $t=5 \mathrm{~min}(B, D, F)$ après addition de 0,2 $\mu \mathrm{M}$ de 4-Bromo-A23187. L'augmentation de la $\left[\mathrm{Ca}^{2+}\right]_{i}$ (A vers $B)$ se traduit par une augmentation de $F_{350}$ (C vers D), corrélée à une diminution de $F_{380}$ (E vers $\left.F\right)$; les images en pseudo-couleurs de la $\left[\mathrm{Ca}^{2+}\right]_{i}(\mathrm{~A}$ et $B$ ) sont obtenues après analyse informatique. (Système d'imagerie IMSTAR, Paris.)

chondries, les lysosomes, les granules de sécrétion ou les endosomes. La forme, libre, partiellement désestérifiée ou estérifiée, sous laquelle la sonde peut être séquestrée, reste débattue et plusieurs mécanismes de séquestration sont incriminés : diffusion passive de la sonde-AM, mécanisme de pinocytose ou transport actif de la forme libre par transporteur anionique. Selon le type de problème suspecté, des solutions variées sont adoptées: une diminution de la température de charge vers $15^{\circ} \mathrm{C}$ peut faciliter l'entrée de la sonde dans la cellule ainsi que sa répartition homogène dans le cytosol ; une augmentation de la température vers $20^{\circ} \mathrm{C}$ peut ensuite améliorer l'efficacité des estérases cellu- laires; l'utilisation d'inhibiteurs de transporteurs anioniques (tel le probénicide) peut enfin permettre de limiter la compartimentation et/ou l'extrusion de la sonde hors de la cellule.

Les spectres de fluorescence du Fura2 et de l'Indol présentent un point isobestique auquel l'intensité de l'émission de fluorescence est indépendante de toute variation de $\mathrm{Ca}^{2+}$ (figure 4). Ce point correspond à une longueur d'onde d'excitation de $360 \mathrm{~nm}$ pour le Fura2 et à une longueur d'onde d'émission de $450 \mathrm{~nm}$ pour l'Indol. La mesure de fluorescence au point isobestique permet de vérifier l'homogénéité de la répartition de la sonde dans la cellule et d'évaluer l'importance de 


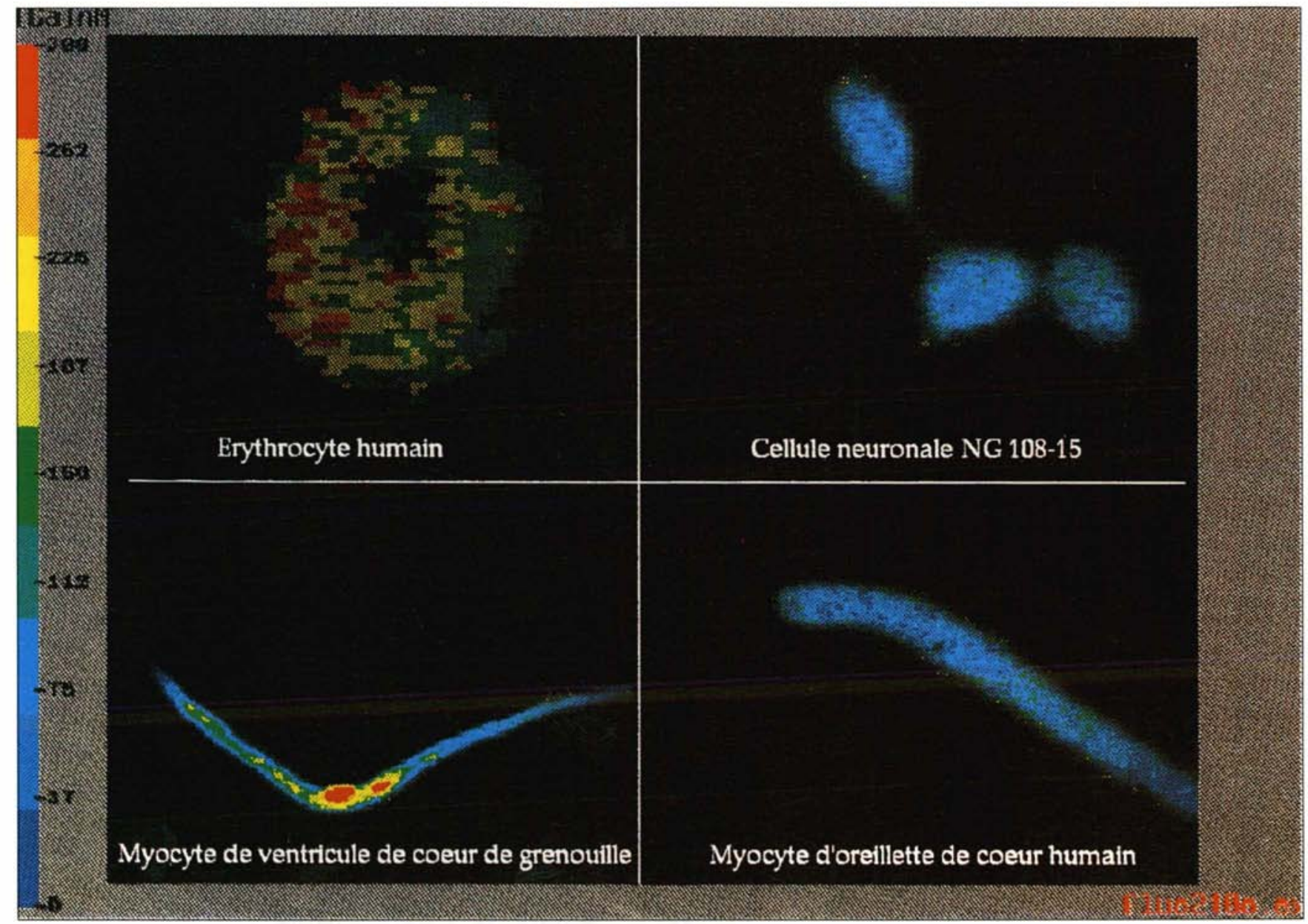

Figure 7. Images en pseudo-couleurs de la $\left[\mathrm{Ca}^{2+}\right]_{i}$ dans différentes cellules chargées en Fura2. Erythrocyte humain (en collaboration avec Y. Beuzard, Inserm U. 91); cellule neuronale NG 108-15; myocyte de cœur de grenouille (en collaboration avec R. Fischmeister, Inserm CJF 92-11); myocyte d'oreillette de cœur humain (en collaboration avec S. Hatem et J.J. Mercadier, Cnrs URA 1159). (Système d'imagerie IMSTAR, Paris.)

l'extinction au cours de l'expérience. Par ailleurs, le choix des longueurs d'onde d'excitation $340 \mathrm{~nm}$ et $360 \mathrm{~nm}$ pour une étude comportant le calcul du rapport de fluorescence à deux longueurs d'onde peut simplifier les problèmes de calibration (puisque, selon l'équation de Grynkiewicz, $\beta=F_{360}$ (en l'absence de $\left.\mathrm{Ca}^{2+}\right) / \mathrm{F}_{360}$ (à concentration saturante de $\mathrm{Ca}^{2+}$ ) aura, par définition, une valeur de 1), mais cela au détriment de la sensibilité de la mesure. L.'Indol est excité à une longueur d'onde et on peut filtrer simultanément par un jeu de miroirs les fluorescences émises à $405 \mathrm{~nm}$ et $485 \mathrm{~nm}$. En revanche, l'emploi du Fura2 nécessite l'utilisation d'un porte-filtres mécanique (chopper) $m / s n^{\circ} 4$ vol. 10, avril 94 pour alterner les deux longueurs d'onde d'excitation (figure 5). A priori donc, l'utilisation de l'Indol semblerait préférable à celle du Fura2 dès que l'on s'intéresse à des cinétiques rapides ou à des cellules dont les fluctuations de $\mathrm{Ca}^{2+}$ modifient la forme ou intensifient le mouvement. A l'aide de l'Indol, il est à l'heure actuelle possible de mesurer une image $\mathrm{Ca}^{2+}$ d'un format digital de $128 \times 128$ pixels, toutes les 40 millisecondes en moyenne. Néanmoins, l'analyse avec de l'Indol suppose l'acquisition simultanée et équivalente des deux images d'émission de fluorescence. Il est donc nécessaire de disposer de deux caméras ou d'un système très perfectionné qui permet la juxtaposition des deux images sur la photocathode d'une seule et même caméra. De fait, l'Indol est encore de nos jours beaucoup moins employé que le Fura2 pour les études d'imagerie sur cellule unique.

Les sondes Mag-Fura2 et Mag-Indol, qui ont les mêmes propriétés spectrales que, respectivement, le Fura2 et l'Indol, ont été développées, à l'origine, pour la mesure du taux intracellulaire de magnésium [21]. Cependant, la faible affinité de ces molécules pour le $\mathrm{Ca}$, ainsi que leur tendance à s'accumuler dans les sites intracellulaires, sont exploitées pour les mesures de variations importantes de Ca, de micro- à millimolaire, qui peuvent se produire localement dans une cellule. En 
outre, leur délai de réponse étant de l'ordre de la milliseconde, leur utilisation est particulièrement adaptée à l'étude des pics calciques dans les cellules excitables.

\section{Les sondes excitables dans le visible}

La nécessité pour les premiers indicateurs calciques d'une excitation dans le domaine de l'ultraviolet limitait les études, du fait de l'optique. La mise sur le marché de sondes mono-excitation/mono-émission dont les spectres de fluorescence sont localisés dans le visible, telles Fluo3 [20], Fura red [21], $\mathrm{Ca}^{2+}$ green et $\mathrm{Ca}^{2+}$ orange[21], a non seulement contribué à développer la mesure du $\mathrm{Ca}^{2+}$ en cinétique rapide, souvent couplée au patch clamp, mais a aussi permis l'ouverture vers de nouvelles technologies utilisant la microscopie confocale et la cytométrie de flux $[22,23]$ (Tableau I et figure 3). La microscopie confocale a donné une troisième et une quatrième dimensions aux études du métabolisme calcique puisqu'elle permet de visualiser les distributions spatiale et temporelle $\mathrm{du} \mathrm{Ca}^{2+}$ intracellulaire en opérant une coupe optique des cellules dans un plan $\mathrm{x}$, y dont la fluorescence de chaque point est analysée individuellement [24]. La cytométrie de flux, qui permet l'analyse statistique d'un grand nombre de cellules (de l'ordre de 300 cellules/s), est, quant à elle, appliquée à l'étude de l'hétérogénéité des réponses calciques qui est rencontrée dans tous les types cellulaires.

Un autre avantage des sondes excitables dans le visible est qu'elles permettent l'utilisation parallèle de molécules "cagées" (ions, nucléotides ou phospholipides) $[6,21,25]$ ou de chélateurs photochimiques (Diazo-2, Diazo-4, Azid-1...) [6, 21] qui sont libérés par éclair UV.

Figure 8. Effet de concentrations croissantes d'isoprotérénol sur la $\left[\mathrm{Ca}^{2+}\right]_{i}$ de cellules embryonnaires de ventricule de cour de poulet. (A) Images numérisées du $\left[\mathrm{Ca}^{2+}\right]_{j}$. (B) Quantification du $\left[\mathrm{Ca}^{2+}\right]_{i}$ au cours du temps sous l'influence de concentrations croissantes d'isoprotérénol.

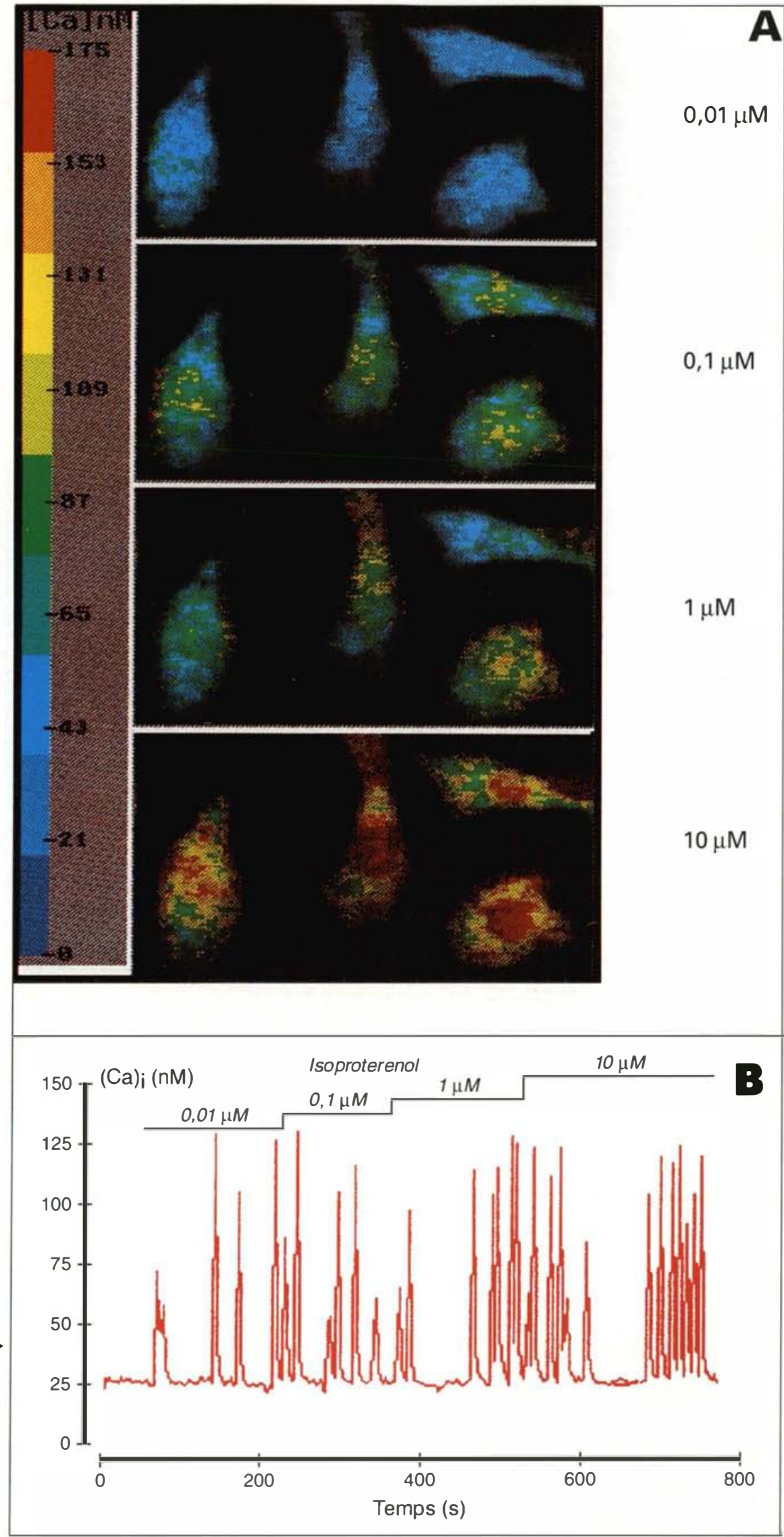

$\mathrm{m} / \mathrm{s} n^{\circ} 4 \mathrm{vol}$. I0, avril 94 


\section{L'évolution vers une sonde fluorescente idéale}

Les qualités requises pour une sonde fluorescente varient selon l'étude envisagée et il est bien évident qu'il n'existe pas de sonde fluorescente idéale dans l'absolu. Le choix d'une sonde doit ainsi résulter d'un bon compromis entre plusieurs critères : (1) spécificité vis-à-vis du $\mathrm{Ca}^{2+}$; (2) affinité (Kd) compatible avec la gamme de concentrations dans laquelle on veut analyser le signal $\mathrm{Ca}^{2+}$; (3) linéarité de la réponse en fonction du $\mathrm{Ca}^{2+}$; (4) vitesse de réponse compatible avec les cinétiques étudiées; (5) faible sensibilité aux modifications de l'environnement ( $\mathrm{pH}$, force ionique...) ; (6) rendement quantique suffisant pour la détection du signal fluorescent ; (7) faible tendance à la liaison non spécifique, à la diffusion, à la compartimentation et à la fuite; des dérivés Dextran des sondes calciques (Fura2-Dextran, Indol-Dextran...) ont été récemment commercialisés par Molecular Probes, qui conservent les propriétés spectrales de ces sondes tout en apportant, du fait de leur taille, une bonne parade à ces problèmes (molecular probes); (8) influence négligeable sur les fonctions cellulaires; et enfin (9) compatibilité avec l'instrumentation dont on dispose.

Il est essentiel de souligner que la qualité des études d'imagerie repose sur le choix d'un équipement informatique performant qui assurera en particulier la digitalisation des signaux analogiques issus de la caméra, et le calcul, pixel par pixel, des valeurs de $\mathrm{Ca}^{2+}$ à partir des mesures de fluorescence, en un temps minimum. Une présentation esthétique des résultats en pseudocouleurs et la convivialité du logiciel sont aussi des qualités bien appréciées par l'utilisateur.

L'inconvénient majeur des nouvelles sondes excitables dans le visible est que leur spectre de fluorescence est de type mono-excitation, monoémission. Des études récentes ont cependant conçu l'utilisation de ces sondes à deux longueurs d'onde par le biais d'une double charge cellulaire de deux indicateurs fluorescents $\mathrm{m} / \mathrm{s} n^{\circ} 4$ vol. 10, avril 94 compatibles, tels le Fluo3 et le Fura red qui sont excitables à la même longueur d'onde $(514 \mathrm{~nm})$. La liaison du $\mathrm{Ca}^{2+}$ au Fluo3 induit une augmentation de son émission de fluorescence à $540 \mathrm{~nm}$, alors qu'elle provoque une diminution de l'intensité de fluorescence du Fura red à $650 \mathrm{~nm}$. L'utilisation d'un mélange de deux indicateurs calciques, en vue du traitement des signaux de fluorescence selon leur rapport, nécessite cependant de s'assurer (1) que ces deux indicateurs sont co-distribués de façon homogène dans la cellule; (2) que tous les éléments structuraux subcellulaires accumulent ou excluent les deux sondes de façon équivalente et enfin (3) que les deux indicateurs présentent les mêmes cinétiques de liaison du calcium. Ce protocole a déjà été exploité pour des études quantitatives en microscopie confocale [22].

Il faut remarquer que le protocole de double charge est aujourd'hui routinier pour l'étude en parallèle de deux paramètres $\left(\mathrm{Ca}^{2+} / \mathrm{pH}, \mathrm{Ca}^{2+} /\right.$ potentiel...).

Les études sur cellules uniques ont rendu possible la mesure cinétique et quantitative des signaux calciques réels, comparés aux signaux moyennés obtenus sur populations cellulaires. Ce sont ces études qui ont révélé le caractère oscillatoire et transitoire des variations de $\mathrm{Ca}^{2+}$. Elles ont aussi permis la caractérisation de régions cellulaires "oscillateurs" où sont initiés les signaux calciques, et la visualisation de propagations de "vagues" de $\mathrm{Ca}^{2+}$ d'une région cellulaire à une autre, ou d'une cellule à l'autre [26].

Des études microfluorimétriques ont aussi été réalisées sur organes entiers et ont permis d'établir des corrélations entre les réponses calciques et l'activité cardiaque, ou la sécrétion biliaire et la synthèse de glucose dans le foie [27].

En conclusion, la technique de microfluorimétrie présente des avantages majeurs par rapport aux anciennes méthodes de dosage du $\mathrm{Ca}^{2+}$ sur cellules : l'incorporation des sondes peut se faire sans traumatisme cellulaire; en outre, cette technique apporte une haute définition spatiale, ainsi qu'une résolution temporelle de l'ordre de la milliseconde, alors que la résolution temporelle des microélectrodes spécifiques du $\mathrm{Ca}^{2+}$ et de la résonance magnétique nucléaire étaient, respectivement, de l'ordre de la seconde et de la minute. Elle est, par ailleurs, adaptable à tous les types cellulaires et d'un abord technique relativement facile comparé à la sophistication des méthodes récentes de localisation ultrastructurale des ions par microscopie électronique avec filtres d'énergie [28].

Mais il ne faut pas pour autant oublier que les percées dans les connaissances du métabolisme calcique ont été conditionnées par le développement d'inhibiteurs et d'activateurs spécifiques, de molécules "cagées" et d'anticorps, qui, grâce à des protocoles ingénieux, permettent l'analyse de chaque composante de l'homéostasie calcique [29-31]

\section{Summary}

Shedding new light on calcium

In the late 1960s, micro injections of giant muscle cells with Aequorin, a $\mathrm{Ca}^{2+}$-triggered bioluminescent protein, gave rise to the first measurements of cytosolic free calcium in living cells. Though partially eclipsed for some years, this old molecule is again up to date thanks to chemistry and molecular biology techniques, for detection of $\mathrm{Ca}^{2+}$ hot spots and measurement of intra-organelles $\mathrm{Ca}^{2+}$ concentrations. In 1982 , Tsien introduced a new generation of fluorescent $\mathrm{Ca}^{2+}$ chelators, derivated from EGTA, and conceived a chemical trick (acetoxymethyl esterification) for loading them by a non-disruptive way into populations of cells of any size. Today, the recent development of highly fluorescent $\mathrm{Ca}^{2+}$ specific probes (Fura2, Indol, and more recently, Fluo3, Fura red, etc.), coupled with advances in microscopy, computer and video imaging technologies, allows a breakthrough in the understanding of $\mathrm{Ca}^{2+}$ homeostasis, making this field one of the most exciting and rapidly evolving of cell biology. 